\title{
Successful treatment of persistent post- operative air leaks following the placement of an endobronchial one-way valve
}

\author{
S. Conforti, M. Torre, S. Fieschi, A. Lomonaco, M. Ravini
}

ABSTRACT: Successful treatment of persistent post-operative air leaks following the placement of an endobronchial one-way valve. S. Conforti, M. Torre, S. Fieschi, A. Lomonaco, M. Ravini.

Treatment of persistent air leaks due to pulmonary resection in pathological conditions of the lung involve a very large spectrum of available methods, from chest drainage and placement of Heimlich valves to surgical repair or pleural decortication. However, in some of these patients surgery may be contraindicated. This report describes an endobronchial approach to the control of marked and prolonged air leaks in four patients using a newly designed one-way airway valve (Pulmonx Corporation; Redwood City, Ca.USA) placed into the segmental bronchus that is the source of air leakage. As the device is a one-way inspiratory airway blocker, it can be used to control persistent air leaks while maintaining the drainage of mucus.

This approach potentially provides an effective nonsurgical and minimally invasive alternative addition to the armamentarium of treatments for patients who suffer with persistent post-operative air leaks where other methods have failed or in frail patients who are categorised as a high surgical risk.

Monaldi Arch Chest Dis 2010; 73: 2, 88-91.

Keywords: Bronchoscopy, Emphysema, Endobronchial stent, Lung lobectomy, Surgery complications.

Thoracic Surgery Department, Niguarda Hospital, Milan, Italy.

Correspondence: Dr. Serena Conforti, Ospedale Niguarda Ca' Granda, Chirurgia Endoscopica del Torace, P.zza Ospedale Maggiore 3, 20100 Milan, Italy; e-mail: serena.conforti@ospedaleniguarda.it

\section{Introduction}

Persistent air leaks can occur after pulmonary resection or in a variety of pulmonary pathological conditions [1]. Treatment and outcome depend on the cause, the quality of the lung and the clinical status of the patients. Standard management involves prolonged chest tube drainage, placement of a Heimlich valve and sometimes pleurodesis [2-4]. Some intractable cases require surgical repair or pleural decortication [5]. However, many patients may be classed as high surgical risks, particularly if they have already undergone thoracic intervention or have another major comorbidity. In these cases we need reliable, less invasive alternatives. A number of procedures are available, including the use of tissue and fibrin glues, sealants, stents, plugs and coils resulting in permanent occlusion of the targeted bronchus $[6,7]$.

The endobronchial valve system (Zephyr EBV) (Pulmonx Corporation; Redwood City, Ca. USA) is designed primarily for endoscopic lung volume reduction in lung emphysema. The initial safety and efficacy of valves in this disease is encouraging and the results from multicentre, prospective, randomised controlled trials are awaited [8-13].

We evaluated the ability of the valve in treating persistent postoperative air leaks and report degree of air-leakage in 4 patients following place- ment of the one-way valve into the segmental bronchus responsible for air-leakage.

\section{Materials and methods}

The valve implant consists of a stent-like, selfexpanding nitinol mesh retainer that secures the EBV in place, and expands to contact the wall of the bronchial lumen to provide a complete seal during inspiration. A medially-facing one-way duckbill valve is mounted in the central part of the retainer. Two sizes of implants were used (4.0 and $5.5 \mathrm{~mm}$ ): the EBV 4.0 designed for bronchial lumens with diameters of $4.0 \mathrm{~mm}$ to $7.0 \mathrm{~mm}$ and the EBV 5.5 for bronchial lumens with diameters of $5.5 \mathrm{~mm}$ to $8.5 \mathrm{~mm}$. The delivery catheter facilitates the placement of the Zephyr valve into the targeted bronchus through the $2.8 \mathrm{~mm}$ diameter working channel of a flexible bronchoscope. The valve is intended to block air from flowing through the air leak. It is also designed to allow mucus to be expelled in order to reduce the likelihood of post-obstructive infection (fig. 1).

Clinical signs and radiological findings (CT scan) are unlikely to identify bronchial subdivisions leading to a leak. To achieve an accurate evaluation, the bronchi were endoscopically examined in detail using the selective bronchial occlusion test [14]. To determine the possible source(s) of the air-leakage, we selectively occluded every 
segmental bronchus by inflating a Fogarty balloon catheter ( 5 or 6 FR balloon wedge pressure) passed through the endoscopic working channel. The chest drainage system was observed during the test. If the air leaks continued, the balloon catheter was placed into another segmental bronchus, monitoring the effect segment by segment until the air leak was halted completely or was considerably reduced. When we identified the leaking bronchus, we estimated the size of the target bronchus with the balloon inflated before selecting a one-way valve of optimal size. (There is a bronchial diameter measurement gauge made of flexible polymer at the proximal end of the distal housing.) The valves can be removed easily if placement is not satisfactory using collapsing forceps through the working channel of the bronchoscope. All procedures were performed in an operating room or bronchoscopy suite with full anesthetic capabilities.

\section{Case Reports}

\section{Case 1}

A 62-year-old man was referred to our clinic after diagnosis of adenocarcinoma of the lung. Severe COPD with emphysema had been diagnosed in 1975 after recurrent bilateral pneumothorax. CT scan of the thorax showed an irregular mass $1.5 \mathrm{~cm}$ in diameter in the left upper lobe, without mediastinal lymphadenopathy. PET scanning confirmed a solitary lung lesion. Pulmonary function tests showed a FEV 1 of $1.03 \mathrm{~L}$ ( $42 \%$ of predicted). A surgical wedge resection of the ventral segment was performed instead of left upper lobectomy. The patient was extubated within 24 hours in the Intensive Care Unit. The post-operative course was complicated by persistent air leakage causing respiratory failure and reintubation for a further 3 days under controlled pressure ventilation. After 7 days an EBV valve was placed in the ventral segment of the left upper lobe. The procedure was performed with a flexible bronchoscope under local anaesthesia, with the collaboration of the patient who coughed when so requested. There was slow but steady progressive re-expansion of the residual parenchyma. The air leak completely stopped within 2 days, allowing the chest tube to be removed on the $4^{\text {th }}$ day. The patient was discharged in good condition and at 6 month the valve was removed without complications.

\section{Case 2}

A 28-year-old woman with Fontan Syndrome (monoventricle) in "situs inversus visceri" underwent cardiac correction surgery when the disease relapsed. Intraoperatively, the surgeon reported that bilateral lung parenchyma was completely adherent to the thoracic wall, the tissue was fragile and the patient required extra-corporeal membrane oxygenation (ECMO) support. After the placement of two chest tubes, the air leakage from the right lung stopped, but a high rate of air leakage persisted on the left. The patient's condition was poor and required ventilator support with $80 \%$ oxygen.

We performed selective intubation using a fiberoptic bronchoscope and continued mechanical ventilation; one tube was placed in the right and another large-diameter tube was placed in the left. Air leakages from the left chest tube remained unabated and continued during all respiratory cycles of the ventilator; the patient was continuously manually ventilated through the right tube because of her precarious respiratory condition. Bronchoscopy was performed through the left side: we performed selective segmental occlusion while monitoring the air leakage. We used general anaesthesia and delivered set volumes of breaths using the digital meter. We were able to demonstrate that the source was in the area of S3 of the left upper lobe where we placed the valve. Immediately after placement, the air leaks stopped as did the bubbling of the chest drain. The patient remained stable in the short term; the chest tube was removed and we maintained mediastinal drainage for purulent effusion. However, she died 2 weeks later due to systemic sepsis resulting from mediastinitis.

\section{Case 3}

A 60-year-old man had bilateral apical bullous emphysema with recurrent left pneumothorax and gradually increasing dyspnea required oxygen therapy. The patient was confined to bed. Initially a left chest tube was placed. Because of continued pneumothorax, after through investigation, the patient was submitted to surgery with large left bullectomy. The post-operative course was complicated by poor general condition, incomplete pulmonary expansion, persistent air leakage and consequent respiratory failure. Bronchoscopy was performed under general anaesthesia with ventilation through a rigid bronchoscope. The source of the air leaks was identified in the apical segment of the upper left lobe. The valve was delivered to the target bronchus and the air leaks halted. During the same procedure we placed another two valves in the upper right lobe bronchus to stop air supply during inspiration, in order to reduce bollous emphysema. The patient was discharged after 2 weeks; at 6 months the valves have not yet been removed. No infectious complications were observed. The CT scan showed good re-expansion of left lung, a great improvement in expansion of the right lung and improved $\mathrm{FEV}_{1}$ value. The patient has a good performance status without oxygen dependence.

\section{Case 4}

A 57-year-old woman had received lower left lobectomy for lung adenocarcinoma at another centre. Intraoperatively the fissure between upper and lower lobe was very fragile because of inflammatory tissue; therefore glue slurry was employed to prevent air leaks. However the post-operative course was complicated by prolonged air leaks that 


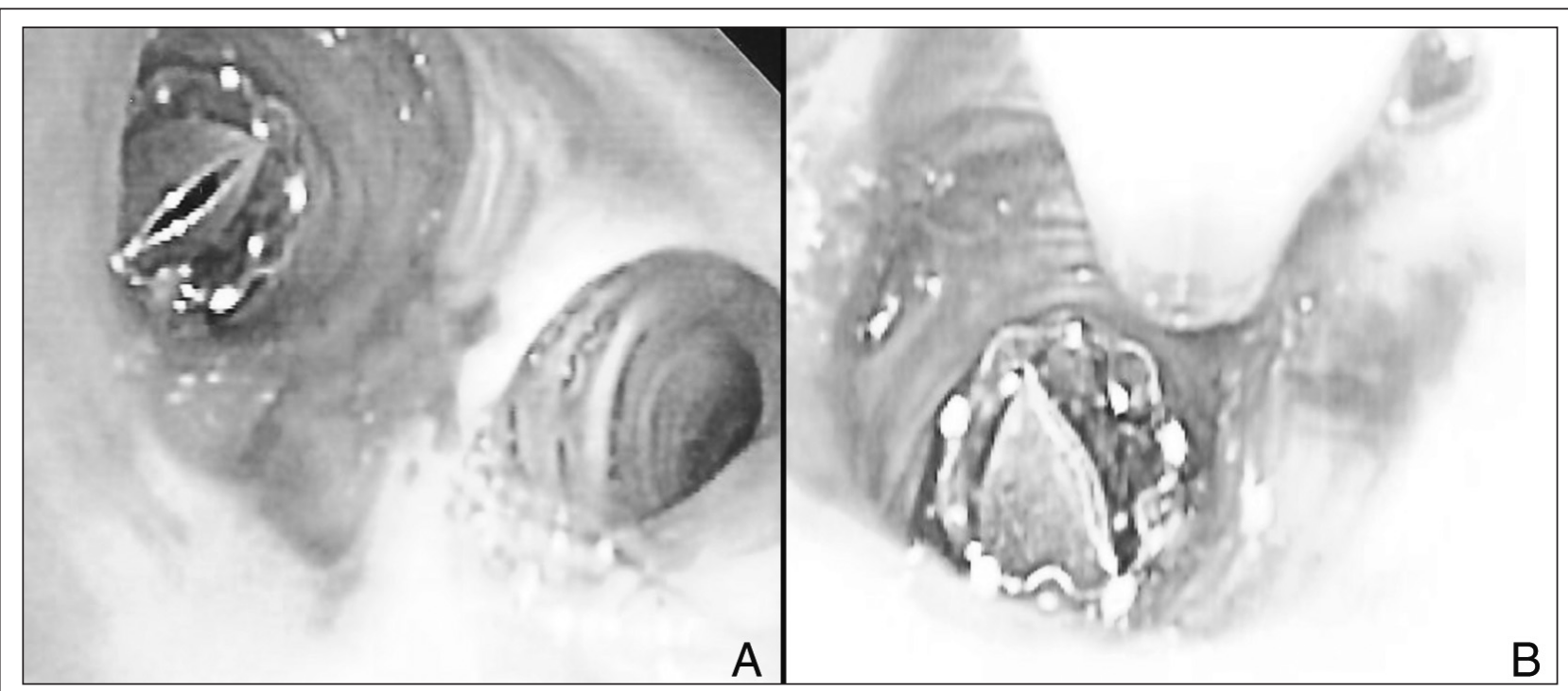

Fig. 1. - Endobronchial one-way valve after placement: A) during expiration; B) during inspiration.

started 48 hours after surgery. A fiberoptic bronchoscope was inserted through a rigid tracheoscope under intravenous sedation and manual ventilation. The valve was placed in the anterior segment $(S 1+2)$ of the left upper lobe. Air leakage stopped within 48 hours. She made a good recovery with improvement of the left pneumothorax. The drainage tube was removed within 4 days. At follow-up, 3 months after the procedure, she had a residual but not clinically significant airspace visible on the chest radiographs. The valve was still in place and no infectious complications or granulations were observed at 6 months on endoscopic follow-up.

\section{Discussion}

We set out to determine whether a fiberoptic endoscopically-inserted one-way valve could be of use in serious cases of post-operative pulmonary air leak. If the lung fails to re-expand after wellmanaged chest tube placement, major airway injury should be suspected, evaluated by bronchoscopy and treated appropriately. A large and persistent air leakage can compromise the clinical condition of critically ill patients, increase respiratory impairment and the risk of infection, delaying weaning from the ventilator. The patients in the present series all had air leaks due to alveolarpleural fistulae, because of communication between the pulmonary parenchyma distal to a segmental bronchus and the pleural space. While surgery may be indicated to treat this complication, it is not always feasible in critical cases. The success rate of a second procedure may be very poor, especially in clinically compromised patients. In the present group we found that the placement of the endobronchial valve device reliably treated the air leak. This approach holds the key to providing an effective nonsurgical endoscopic, which is a less invasive alternative to the management of persistent air leakage. The EBV works like a Heimlich valve, allowing air outflow and mucus clearance, but preventing air inflow. No major complications were encountered in this series. The valve was well tolerated and can be removed, when the tear on the lung surface is sealed and we can reasonably expect no recurrence. No infectious complications and no pneumonia or atelectasis (even in case 3) were observed: all patients were treated with a short term of broad-spectrum antibiotics (prophylactic intravenous cefazolin). The EBV have a different mechanism to prevent secretion accumulation than the endoscopic Watanabe spigot [14], allowing mucus to be successfully expelled. Watanabe spigot is in fact a silicone endobronchial blocker frequently used in past in the treatment of bronchopleural fistula or massive focal hemoptysis. This valve is cheaper than Zephyr type but there are two important differences: first the Watanabe spigot is rigid and, like silicone stents, not expansible; second, the spigot completely blocks the air even in inspiration phase; indeed it is a complete occlusive plug. EBV is conformed, as a self expanding stent able to blocks air only during inspiration but allows air flow during expiration. For these reasons the Zephyr valves are more useful in this kind of patients.

The clinical progress of these patients was at least influenced by the condition that led to the occurrence of the leaks. We ascribe the death of patient 2 to a pre-existing systemic infection and do not consider it to be related to the valve insertion.

The endoscopic procedure can be performed under local or general anaesthesia, through a rigid bronchoscope or through an orotracheal tube with the help of ventilation or the patient coughing. Anaesthesia and access approaches depended on patient status.

In relation to Case 3, we placed two EBV in order to reduce at the same time controlateral bollous emphysema. The EBV were primarily designed for the treatment of heterogeneous emphysema. Lung volume reduction surgery and bulla resection may be a palliative option when medical 
therapy has failed; however only a limited number of patients are suited. In these cases the endobronchial approach could provide palliation with less risk than surgical treatment, removing the least hyperinflated lung in order to relieve dyspnea and improve quality of life [8-13].

In conclusion, these results suggest that the endoscopic placement of EBV is a valid option to help resolve difficult air leakage situations, reducing the length of hospitalisation and health-care costs [1-3]. Several investigators [15-19] have reported the use of this valve in the management of persistent air leaks following many pulmonary diseases; they describe complex clinical cases in which nonsurgical intervention was most appropriate. In our report we focused our attention on the treatment of only postoperative critical cases. Endobronchial blockage appears to be a possible salvage procedure when no other treatment is available. Pending confirmation of success in other cases, as well long-term safety and effectiveness data, bronchoscopic EBV treatment may became an attractive alternative in such patients. The future role of EBV in the treatment of persistent air leak will depend in fact on the balance between efficacy, safety and cost.

Acknowledgements: The authors are indebted to Prof. Patrick Barron of the International Medical Communications Center of Tokyo Medical University for his review of this manuscript.

\section{References}

1. Rice TW, Okereke IC, Blachstone HH. Persistent air leaks following pulmonary resection. Chest Surg Clin North Am 2002; 12: 529-39.

2. Cerfolio RJ, Tummala RP, Holman WL, et al. A prospective algorithm for the management of air leaks after pulmonary resection. Ann Thorac Surg 1998; 66: 1726-31.

3. Cerfolio RJ, Bass CS, Pask AH, et al. Predictors and treatment of persistent air leak. Ann Thorac Surg 2002; 73: $1727-30$

4. Cerfolio RJ, Bass C, Katholi CR. Prospective randomized trial compares suction versus water seal for air leaks. Ann Thorac Surg 2001; 71: 1613-7.
5. Puskas JD, Mathisen DJ, Grillo HC, et al. Treatment strategies for bronchopleural fistula. J Thorac Cardiovasc Surg 1995; 109: 989-96.

6. Lin J, Ianettoni MD. Closure of bronchopleural fistulas using albumin-glutaraldehyde tissue adhesive. Ann Thorac Surg 2004; 77: 326-8.

7. Watanabe S-I, Watanabe T, Urayama H. Endobronchial occlusion method of bronchopleural fistula with metallic coils and glue. Thorac Cardiovasc Surgeon 2003; 51: $106-8$

8. Venuta F, Rendina EA, De Giacomo T, Anile M, Diso D, Andreetti C, Pugliese F, Coloni GF. Bronchoscopic procedures for emphysema treatment. Eur J Cardiothorac Surg 2006; 29: 281-7.

9. Venuta F, De Giacomo T, Rendina EA, Ciccone AM, Diso D, Perrone A, et al. Bronchoscopic lung volume reduction with one-way valves in patients with emphysema. Ann Thorac Surg 2005; 79: 411-7.

10. Strange C, Herth F, Kovitz KL, McLennan G, et al. Design of the Endobronchial Valve for emphysema palliation trial (VENT): a non-surgical method of lung volume reduction. BMC pulmonary medicine 2007; 7: 10.

11. Toma TP, Hopkinson NS, Hillier J, et al. Bronchoscopic volume reduction with valve implants in patients with severe emphysema. Lancet 2003; 361: 931-3.

12. Innes $\mathrm{Y}$, Wan P, Toma TP, Geddes DM, Snell G, Williams T, et al. Bronchoscopic lung volume reduction for end-stage emphysema: report on the first 98 patients. Chest 2006; 129: 518-526.

13. Hopkinson NS, Toma TP, Hansell DM, Goldstraw P, Moxham J, et al. Effect of bronchoscopic lung volume reduction on dynamic hyperinflation and exercise in emphysema. Am J Respir Crit Care Med 171; 453-460.

14. Watanabe Y, Matsuo K, Tamaoki A, et al. Bronchial occlusion with endobronchial Watanabe spigot. J Bronchol 2003; 10: 264-7.

15. Snell GI, Holsworth L, Fowler S, et al. Occlusion of a broncho-cutaneous fistula with endobronchial one-way valves. Ann Thorac Surg 2005; 80: 1930-1932.

16. Mitchell KM, Boley TM, Hazelrigg SR. Endobronchial valves for treatment of bronchopleural fistula. Ann Thorac Surg 2006; 81: 1129-1131.

17. Ferguson JS, Sprenger K, Van Natta T. Closure of a bronchopleural fistula using bronchoscopic placement of an endobronchial valve designed for the treatment of emphysema. Chest 2006; 129: 479-481.

18. Toma TP, Kon OM, Oldfield W, et al. Reduction of persistent air leak with endoscopic valve implants. Tho$\operatorname{rax} 2007$; 62: 830-833.

19. Anile M, Venuta F, De Giacomo T, et al. Treatment of persistent air leakage with endobronchial one-way valves. J Thorac Cardiovasc Surg 2006; 132: 711-712.

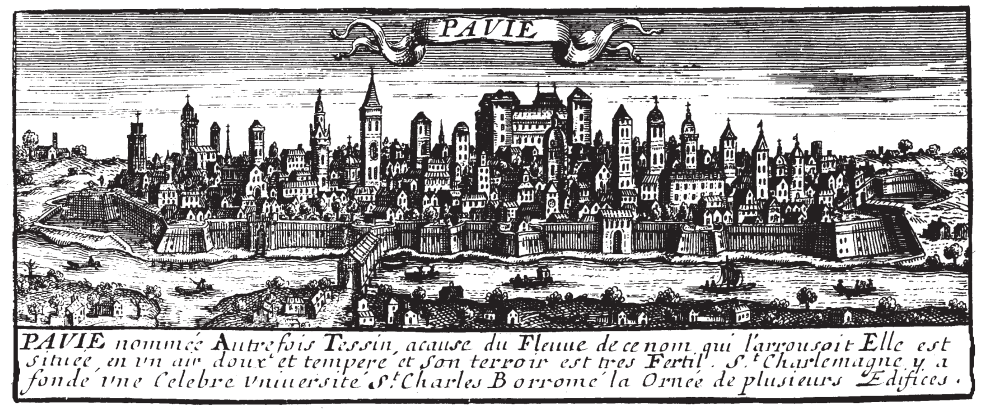

